\title{
Novel concept in cancer metastases operated by growth factors and somatic stem cells
}

\begin{abstract}
Cancer metastasis represents one of the fundamental and unique biological characterizations of malignancy. It is a hot issue in cancer research since; the major cancer problem is mainly due to metastasis. Therefore, studying metastasis mechanisms and routes for cancer cell spreading could be tremendous importance for the control and management of malignancy. The process of metastasis dissemination of malignant tumor cells to form secondary foci at distant sites is a complex and dynamic process. Direct proof of causal roles in tumor growth, migration, invasion, angiogenesis and metastasis has been shown by up-regulation of growth factors in malignant tumor cells. In addition to, Stem cells localized in different organs possess crucial role in the incidence of neoplastic transformation that could occur in these organs.
\end{abstract}

The present article could be a novel concept for cancer metastasis. It highlights the coordination of the transforming growth factors $(\alpha \& \beta)$, vascular endothelial growth factors and somatic Stem cells in enhanced cancer metastasis.

Keywords: cancer metastasis, vascular endothelial growth factor, transforming growth factors (a and b types), stem cells
Volume I Issue 2 - 2014

\author{
Motawa E EL-Houseini \\ Department of Tumor Biology, Cairo University, Egypt
}

Correspondence: Motawa E EL-Houseini, Department of Tumor Biology, National cancer Institute, Cairo University, I 1796 Cairo, Egypt, Tel 002023635083,

Email motawa_matter@yahoo.com

Received: April 22, 2014 | Published: September 02, 2014
Abbreviations: VEGF, vascular endothelial growth Factor; $\mathrm{TGF} \alpha$, transforming growth factor alpha; TGF $\beta$, transforming growth factor beta; EGF, epidermal growth factor; PDGF, platelet derived growth factor; IGF, insulin-like growth factors; PLGF, placental-like growth factor; NPs, neuropilins; FLT, fms-like tyrosine kinase; TIMP, tissue inhibitor metallo proteinase; EMT, epithelial-mesenchymal transition; MMP, matrix metallo proteases; IL, interleukin

\section{Cell signaling cascade pathway}

An understanding the mechanisms responsible for the control of normal proliferation and differentiation of the various cell types is a important issue. This allows greater insight into the abnormal proliferation of malignant cells in the human biological system. ${ }^{1,2}$

Particular attention is focused on the signals that act as positive and negative regulators of the cell proliferation. Such signals secreted polypeptides that provide information essential for co-ordinated pattern of cell- cell communication. ${ }^{3}$

A signaling pathway begins with the arrival of a chemical signal - such as a hormone or growth factor - at the cell surface. Growth factor signals are propagated from the cell surface, through the action of transmembrane receptors, to intracellular effectors that control critical functions in human cancer cells, such as differentiation, growth, angiogenesis, and inhibition of cell death and apoptosis. ${ }^{4}$ The receptors, structures sticking out of the cell membrane, receive these incoming signals. The signal encounters and binds to its matching receptor. A second receptor protein joins in, making the growth factor fit like a key in a lock. The binding of the growth factor causes the receptors to change shape. This change in the protein will be conducted through the membrane and into the cell's interior - the cytoplasm.

Many signaling pathways ultimately pass messages to the nucleus of a cell. The signal is passed to yet another messenger. These messenger proteins are known as kinases, enzymes with the ability to activate other proteins through the addition of phosphate groups. This protein travels to the nucleus past cellular organelles such as the mitochondria and the network of membranes known as the endoplasmic reticulum.

The newly made proteins leave the endoplasmic reticulum wrapped in a layer of membrane called a vesicle. They travel toward the Golgi apparatus where the proteins are modified and sorted for transport. The proteins are transported through the cytoplasm and delivered to where they are needed. The released proteins will signal surrounding cells, or, in some pathways to cancer, will coax this cell into further action. ${ }^{4-7}$

In cancer cells, these signaling pathways are often altered and results in a phenotype characterized by uncontrolled growth and increased capability to invade surrounding tissue. Therefore, these crucial transduction molecules represent attractive targets for cancer therapy. In addition, the signaling pathway plays a key role in tissue homeostasis and organ size control by regulating tissue specific stem cells. Moreover, it plays a prominent role in tissue repair and regeneration. So, dysregulation of it is associated with cancer development. $^{7}$

There are three types of signaling that are operating at the molecular basis of the growth factor functioning. The latter regulates cell proliferation and survival in response to cell - cell and cell- matrix interactions. The type of cell signaling can be classified as follow
i. Autocrine type (A)
ii. Paracrine type (B)
iii. Endocrine type $(\mathrm{C})$
iv. Direct cell-cell signaling (D)

The first system of signaling (A), in which the cell type that synthesizes the growth factors expresses receptors that can elicit a 
cellular response to these factors. The second system (B), that operates between cells in close proximity. In this type of signaling, one cell synthesizes and secretes growth factors for which, the appropriate receptors that are expressed on another nearby cell. In the third system of signaling (C), growth factor synthesis occurs in a specific tissue or organ often at some distance from target cells that express the appropriate receptors. The last one (D) type occurs via a legend exists on the cell surface and its receptor on the responding cell.

The growth factors are regulated by specific releasing factors that, control their storages and secretions to the blood. It is apparent that combination of these types of cell signaling can provide formidable regulatory systems so as to control cell proliferation. ${ }^{8}$

\section{Growth factors and cancer}

Many biological processes including wound healing, development and carcinogenesis involve defined patterns of cellular growth and differentiation. Rates of proliferation and pathways of differentiation are regulated and dependent upon precise and coordinated networks of intercellular communication. The latter may involve direct cellto-cell contact, with junction elements permitting transfer of signals between cells. Thus cells may interact through the extracellular matrix which surrounds all cells in vivo and whose structure and composition is determined by tissue requirements. Alternatively, cells may also communicate indirectly by means of soluble factors which are secreted by a cellular type and diffuse through the extracellular matrix to reach target cells lying at varying distances from the source. ${ }^{9}$

Integration of these extracellular signals underlies tissue homeostasis. Although departure from homeostasis and tumor initiation are instigated by oncogenic mutations rather than by growth factors, the latter are the major regulators of all subsequent steps of tumor progression, namely clonal expansion, invasion across tissue barriers, angiogenesis, and colonization of distant niches. Also, growth factors are frequently involved in evolvement of resistance to therapeutic regimens, which extends the roles for polypeptide factors to very late phases of tumor progression and offers opportunities for cancer therapy. ${ }^{10}$

Moreover, growth factors can also influence normal cell differentiation and constitutive activation of growth -promoting pathways in cancer cells that can modulate the cell phenotype. ${ }^{11}$

Malignant transformation might result from excessive production of growth promoting polypeptides by cancer cells themselves, which both secrete and respond to these factors. Subsequently, several isolated tumor cell types were shown to release a variety of growth stimulatory polypeptides into their conditioned media, and to possess corresponding receptors for these growth stimulatory polypeptides.

Furthermore, the demonstration of growth inhibitory substances in addition to stimulatory peptides in conditioned media of cancer cells led to a revised autocrine hypothesis. This embodied the concept that "malignant transformation may be the result not only of excessive production, expression and action of positive autocrine growth factors, but also of the failure of cells to synthesis, express or respond to specific negative growth factors they normally release to control their own growth". ${ }^{12}$

Therefore some growth factors are stimulatory, while others are inhibitory to epithelial proliferation. Growth factors are produced and secreted locally by many cell types, and they bind to tyrosine kinase receptors on the cell membrane. This activates the membrane protein through the addition of phosphate groups, which in turn activates proteins, such as kinases, in the cytoplasm. Several other proteins may be involved in the cascade, ultimately activating one or more transcription factors. The activated transcription factors enter the nucleus where they stimulate the expression of the genes that are under the control of these factors as shown in Figure 1.

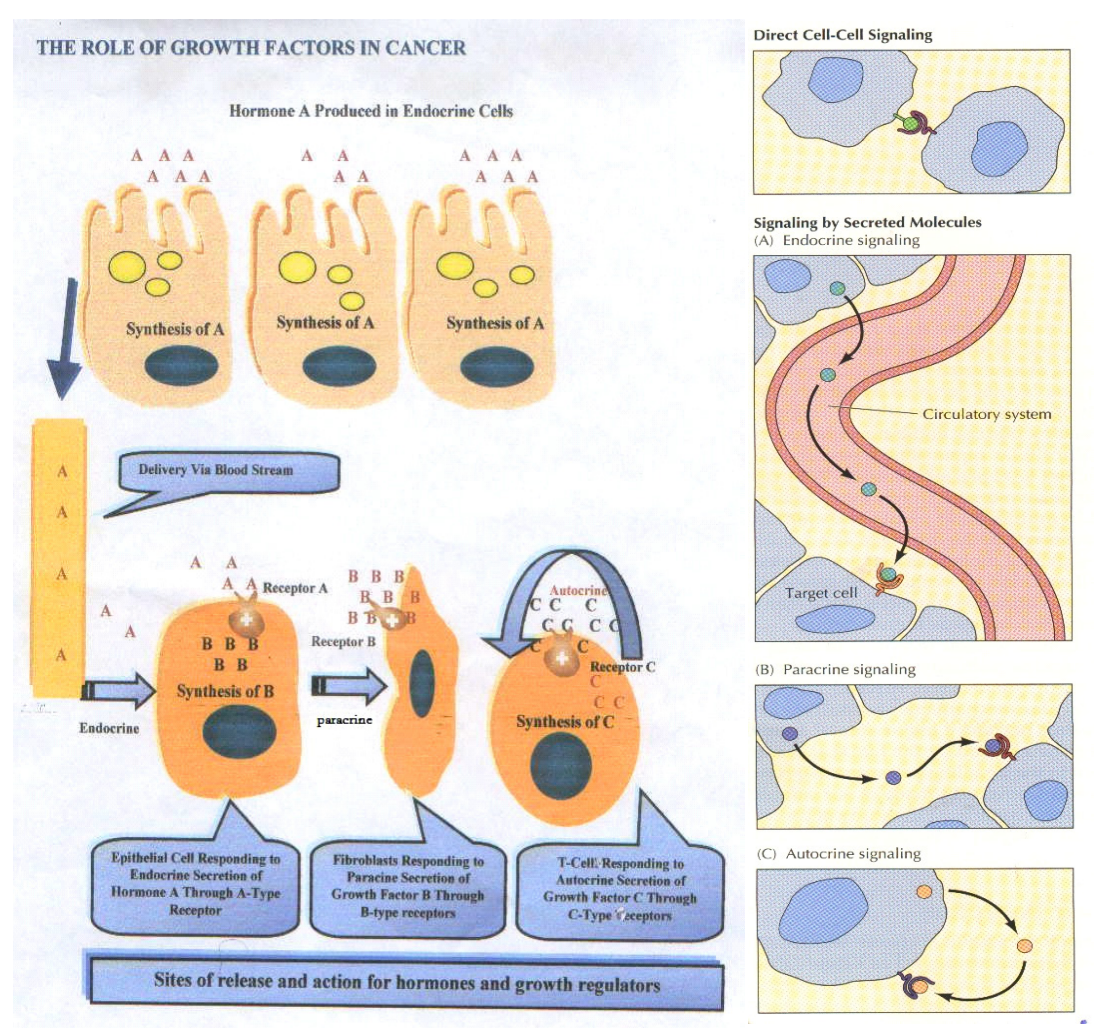

Figure I Type of cell signaling that, are operating in human biological system .mainly autocrine, paracrine and endocrine. 
Growth factors can function either in an autocrine capacity, whereby they act upon their cell of origin, or can interact with adjacent cells of a similar or different type in a paracrine action. The local microenvironment of a cell contains a pool of positive and negative growth factors, which may be functioning in either an autocrine or paracrine manner. It is the balance of these growth factors which determines the polarity and intensity of the effective signal delivered to epithelial cells. There may be an excess of stimulatory growth factors derived either from epithelial cells themselves, or stromal cells. Conversely, the production of negative growth factors which normally serve to keep cell proliferation in check may be deficient. Breakdown of these loops could also result from an abnormal response of target cells to normal levels of growth factors. ${ }^{13,14}$

Much of the experimental work on growth factors and cancer pertains to breast malignancy, with various established breast cancer cell lines have been employed for in vitro investigation of growth factor profiles. The majority of growth factors studied in detail are short polypeptides which have been isolated from the conditioned media of breast cancer and certain other cell lines. These include transforming growth factor alpha (TGF $\alpha$ ), transforming growth factor beta (TGF $\beta$ ), epidermal growth factor (EGF), platelet derived growth factor (PDGF) and insulin-like growth factors (IGF).

\section{Vascular endothelial growth factors}

Vascular endothelial growth factor (VEGF) is one of the most important growth and survival factors for endothelium. VEGFs regulate both vasculogenesis and angiogenesis. The family consists of five glycoproteins, VEGFA (VEGF is called also VEGF-A), VEGFB, VEGFC, VEGFD, and PIGF (placenta-like growth factor). ${ }^{15}$ VEGF is a homodimeric heavily glycosylated protein of $46-48 \mathrm{kDa}(24 \mathrm{kDa}$ subunits). The subunits are linked by disulfide bonds. The human factor occurs in several molecular variants of amino acids arising by alternative splicing of the mRNA. The splice forms of VEGF differ in biological properties such as the receptor types. The 165 amino acid form of the factor (VEGF-165) is the most common form in most tissues. Multiplicity also characterizes the respective surface receptors, co-receptors like neuropilins (NPs) and proteogly-cans, as well as the downstream signaling pathways. ${ }^{16}$

VEGF receptors: Three receptors of tyrosine kinases have been described as putative VEGF receptors and have been shown to bind VEGF with high affinity.

The VEGF family members bind to at least one of the three known VEGFRs, namely VEGFR-1 (FLT-1; fms-like tyrosine kinase-1), VEGFR-2 (FLK-1 or KDR; kinase-insert-domain-containing receptor), and VEGFR-3 (FLT-4; fms-like tyrosine kinase-4). VEGFA binds to VEGFR-1 and VEGFR-2, whereas VEGFB and PIGF bind exclusively to VEGFR-1. VEGFR-2 seems to mediate most known cellular responses to VEGF and has much higher intracellular signaling intermediates than VEGFR-1. ${ }^{17}$ Unlike VEGFR-3, which is largely restricted to lymphatic endothelial cells, both VEGFR-1 and VEGFR-2 are expressed in vascular endothelial cells, as well as monocytes, macrophages (VEGFR-1), and hematopoietic stem cells (VEGFR-2). Importantly, expression of VEGFR-1 and VEGFR-2, as well as the co-receptors NP1 and NP2, has been detected on subsets of solid tumor cells, and according to a recent study activation of VEGFR-1 in breast cancer cells supports their growth and survival. ${ }^{18}$

VEGFRs control angiogenesis by simultaneous signaling through several intermediates: cell proliferation and vasopermeability are stimulated by the protein kinase $\mathrm{C}$ pathway. Other than the proangiogenic effects, VEGF exerts effects independent of vascular processes, such as autocrine effects on tumor cell function (i.e., survival, migration, invasion), immune suppression, and recruitment of bone marrow progenitors. The latter may dictate organ-specific tumor spread by homing to tumor-specific premetastatic sites and forming clusters that provide a permissive niche for incoming tumor cells. ${ }^{19}$

Biological role of VEGF: A well-documented functions of VEGF are; 1- Mitogenesis, angiogenesis, and endothelial survival. 2- Effects of VEGF on bone marrow cells and hematopoiesis. 3- Enhancement of vascular permeability and hemodynamic effects. ${ }^{20-24}$

The action of VEGF is essential to maintain proper endothelial and vascular function. VEGF stimulates virtually all aspects of endothelial function. This includes the stimulation of proliferation, migration, nitric oxide production and release from endothelial cells, and also the permeability of the endothelial cell layer.

The fact that solid tumor growth and metastasis is angiogenesisdependent suggests a potential value of blood and tissue angiogenic markers as prognostic and survival determinants. Angiogenesis which is of great importance for tumor growth and nutrition, is preferentially mediated by the cytokine vascular endothelial growth factor (VEGF) that functions as the most potent angiogenic protein. ${ }^{17,23}$

\section{Transforming growth factor alpha (TGF- $\alpha$ )}

Transforming growth factor alpha (TGF alpha) is one growth factor that has growth of breast cancer cells.

Transforming Growth factor- $\alpha$ is a growth factor produced by several different primary tumors and cancer cell lines that has been circumstantially implicated in regulating the autocrine mechanism. TGF- $\alpha$ is able to cooperate with other growth factors, such as TGF- $\beta$, platelet-derived growth factor and basic fibroblast growth factor, to reversibly induced transformed phenotype in non-transformed cells. ${ }^{25,26}$ The enhanced expression of rat TGF- $\alpha$ gene can lead to the full transformation of rat fibroblasts and partially transformation of rat kidney cells. ${ }^{27}$

TGF- $\alpha$ is an intermediary in transformation of human mammary epithelial cells by an activated c-Ha-Ras gene, but not by the c-neugene. It has been demonstrated that, over expression of this growth factor was able to transform immortalized human mammary epithelial cells which also expressed a sufficient complement of functional EGF receptors (Figure 2). ${ }^{28,29}$

\section{Transforming growth factor Beta (TGF- $\beta$ )}

TGF- $\beta$ exists in three isoforms (TGF- $\beta 1$, TGF- $\beta 2$, and TGF- $\beta 3$ ), but the extended superfamily includes more than 30 additional cytokines, classified into several subfamilies. Some cells secrete and respond to TGF- $\beta$ in an autocrine manner. This cytokine induces a cytostatic effect on many epithelial cell types, and it is also able to control proliferation, differentiation, and programmed cell death in most other cell types because the receptors are widely expressed in derivatives of all three embryonic cell layers.

TGF- $\beta$ is an inducer for the expression of the matrix metalloproteases (MMP-2 and MMP-9) and the down regulation of protease inhibitor (TIMP) in tumor and endothelial cells. Receptor signaling is regulated both positively, by metalloproteinases that cleave a latent form of the ligand, and negatively, by a cell surface antagonist called Bambi. ${ }^{30}$ The receptors play an important role in apoptosis by signaling through 
the SMAD pathway. TGF- $\beta$ can also trigger apoptosis through deathdomain-associated protein $6 .{ }^{31}$

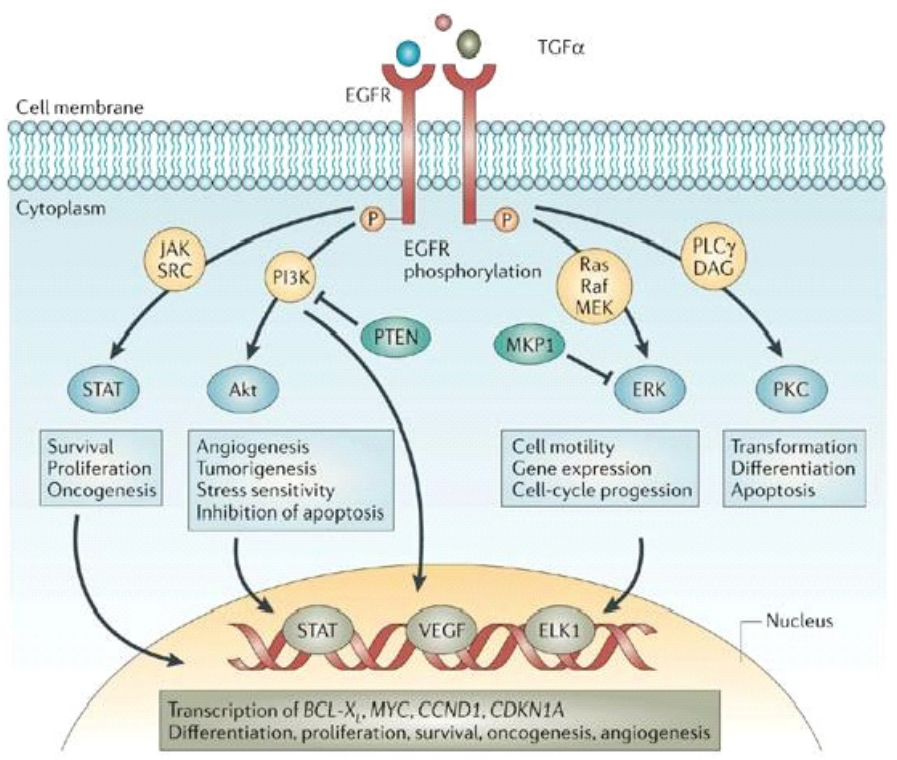

Figure 2 Cascade pathway of TGF- $\alpha$.

In normal cells, TGF $\beta$ is a potent growth inhibitor. However, it is well known that, TGF- $\beta$ is pro-oncogenic agent and that; this growth factor was required for the metastases in most tumor types. ${ }^{20,21}$ Under normal physiological conditions, TGF- $\beta$ prevents the ability of cells to progress through the cell cycle, and it stimulates apoptosis or differentiation. During tumor genesis, however, genetic and epigenetic events can convert TGF- $\beta$ into a tumor promoter. TGF- $\beta$ activates epithelial-Mesenchymal transition (EMT) through activation of both the canonical (i.e., SMAD2/3-dependent) and the noncanonical (i.e., SMAD2/3-independent) pathways. On the other hand, several lines of evidence indicate that the noncanonical TGF- $\beta$ signaling also plays an essential role in mediating TGF- $\beta$ stimulation of EMT, invasion, and metastasis. ${ }^{32}$

The oncogenic activities of TGF- $\beta$ are mediated by dysregulated autocrine and paracrine signaling networks involving epithelial, fibroblast, endothelial, and immune cells that subtly promote tumor angiogenesis and metastasis as well as inhibit host immunosurveillance. The pro-angiogenic functions of TGF- $\beta$ have been linked to its ability to regulate the expression and/or activities of other angiogenic factors, such as FGF and VEGF. ${ }^{33}$ Likewise, stromal fibroblasts may determine whether TGF- $\beta$ suppresses or promotes tumor formation.

Transgenic expression of activated TGF $\beta 1$ in mouse skin epidermis increased the conversion to carcinoma. Also, tumor formation and metastasis to bone was shown to depend on intact TGF- $\beta$ RII. ${ }^{34,35}$

TGF- $\beta$ can control the expression of the stem cell antigenCD34 and under certain circumstances it prohibits the cell differentiation. Therefore, TGF- $\beta$ could preserve the self- renewal of the stem cell. ${ }^{36,37}$

The mechanism thought to contribute the most to the escape from immuno-surveillance is the secretion of cytokines by cancer cell that inhibits immune response. Such immuno-suppressor factors include prostaglandin, IL10 and TGF- $\beta$, the latter is considered to be the most potent one.

Once TGF- $\beta$ becomes activated, it can bind to RII receptor which then phosphorylates type I receptor. The next step in the signal transduction pathway is the phosphorylation of downstream elements Smad $2 \& 3$ that are phosphorylated by TGF- $\beta$ RI and form complexes with Smad 4. Activated Smad complexes enter the nucleus where they regulate transcription of target genes through physical interaction and functional cooperation with DNA-binding transcription factors however, Smad $6 \& 7$ inhibit the previous signal pathway by directly interacting with TGF- $\beta$ RI and preventing Smad $2 \& 3$ phosphylation. ${ }^{38,39}$

It has been reported that, long term treatment of TGF- $\beta$ on cells of rat WB-F 344 could lead to increase their self-renewal capacity and tumorigenicity in NOD-SCID mice. Moreover, in human HCC, it was observed that, a positive correlation between TGF- $\beta$ level and tumor initiating cell markers expression (Figure 3).

\section{Signaling Pathway of TGF- $\beta$}

SIGMA-ALDRICH

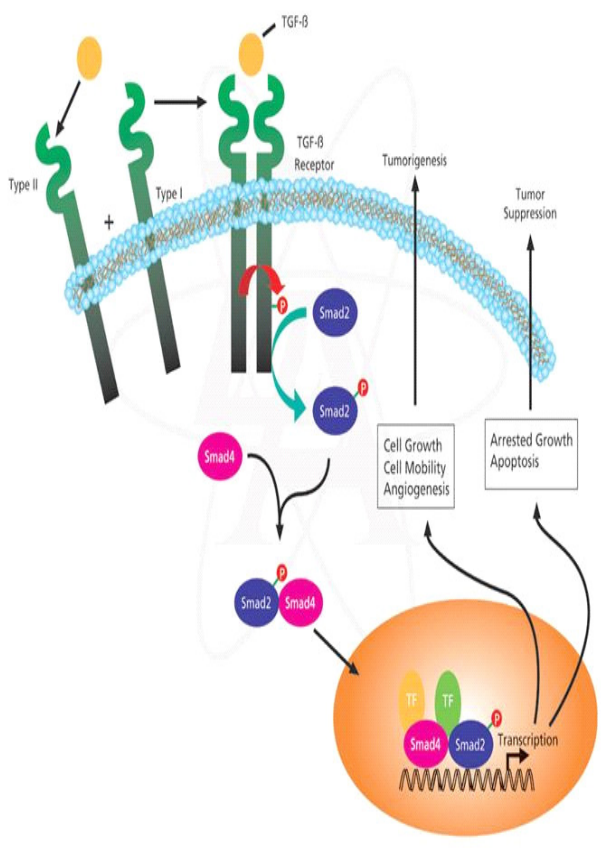

Figure 3 The transforming growth factor $B$ (TGF- $\beta$ ) signaling pathway.

\section{Stem cells}

There are two types of stem cells; embryonic stem cells and somatic (adult) ones; the latter are involved in the regeneration of tissues during the life time of the individual. As a matter of fact stem cells are believed to be present in all tissues so as to replace matured and dying off cells. ${ }^{40,41}$

There are two defining features of stem cells first their ability to self- renewal second their ability to give rise to differentiated cell types of one or more cell lineages. Up on stem cell division, one daughter cell maintains the characteristic of a stem cell including the ability to self-renewal and the other daughter cell shows characteristics of commitment towards differentiation, the feature of self-renewal is shared with cancer cells. ${ }^{42}$

This common feature has led to two proposals for the relevance of stem cell biology to carcinogenesis. The first proposal is that selfrenewal provides increased opportunities for carcinogenic changes to occur. The second proposal_suggested that altered regulation of self- 
renewal directly underlies carcinogenesis. Stem cell must maintain a balance between self-renewal and differentiation the relevance of the stem cell biology to carcinogenesis is that, the loss of this balance could lead to unregulated self-renewal, a hallmark of cancer cells. Therefore, cancer can initiate either in stem cell that has lost regulation of self-renewal or in a differentiated cell that has obtained the ability to self-renewal that is supported by the identification of cancer stem cells (Figure 4). ${ }^{43,44}$

\section{Stem Cell Characteristics}

\section{1) Ability to self renew, to make exact copies of itself through cell division}

\section{2) Ability to develop into different types of mature cells}

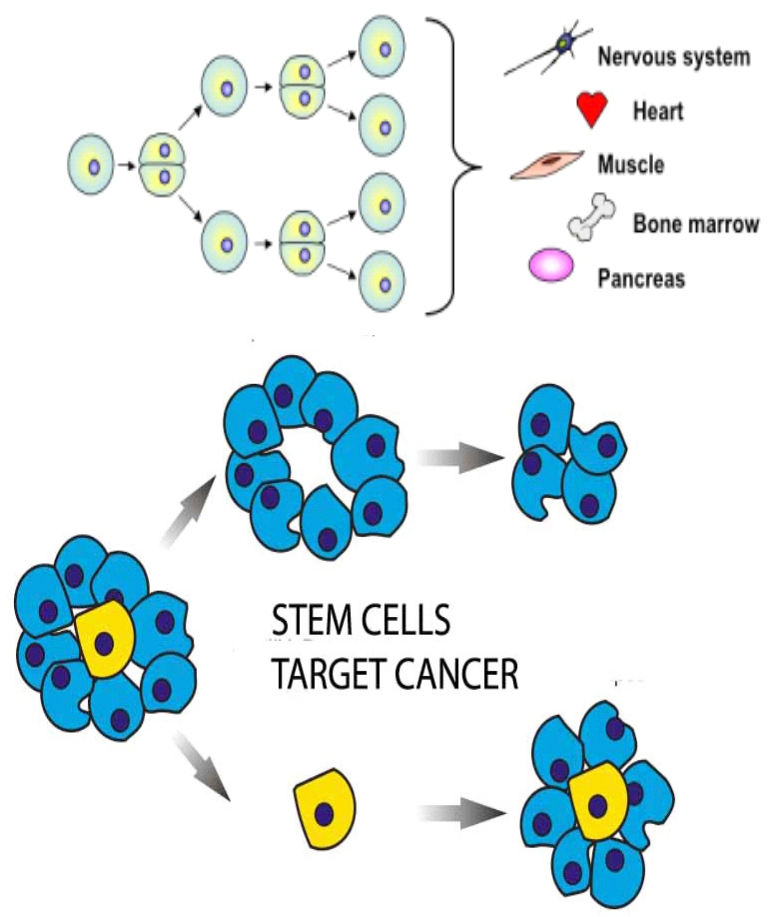

Figure 4 Stem cells are proginators for the formation of different organs. They could also be sites for neoplastic transformation that occurs in these organs.

\section{Phenomenology of cancer metastasis}

It is the process by which cancer cells migrate from a primary site to other parts of the body via blood or lymphatic vessels. It is the fundamental difference between a benign and malignant growth and represents the major clinical problem of cancer. A primary malignant tumor can be surgically removed relatively easily, whereas once multi-metastasis sites could be established throughout the body that are practically impossible to be removed. The process of cancer metastasis comprises a set of diverse events predominantly considered in control the spread of malignant cells to distant site from the primary focus as well as their ability to establish secondary malignant tumor growth independent of the primary one. Cancer metastasis is carried out at different levels; from tumor- host interactions, through factors involved in de-adhesion, migration and colonization of distant sites, to the molecular genetic factors traceable to specific and general behavioral pattern of various metastasizing malignant tumors. ${ }^{45}$ The understanding of the intricate mechanisms underlying the multistage phenomena of metastases is still incomplete. In the malignant tumor micro-environment, paracrine type of signaling is not functioning well. However, both autocrine and endocrine systems of signaling are still actively acting in cancer cell population.

The biological heterogeneity of primary and metastasis tumors extend to a variety of phenotypic characteristics such as receptor status, antigenic profiles, sensitivity to chemotherapeutic agents and many others. ${ }^{46}$ The specificity of intercellular interactions in the process of metastasis is truly amazing, particularly considering that, targeted metastasis spread occurs only when the transplants are placed in specific organ locations, supporting the so-called seed and soil hypothesis. The selective organ and intra-organ specific homing of metastasizing cells of various malignant cells cannot be explained solely on the basis of blood vessel and lymphatic topography. ${ }^{47}$

This issue in cancer metastasis is still to be clarified. Therefore, the present hypothesis is going to throw a light on novel concept in organ selectivity of metastasis.

\section{Novel hypothesis for cancer metastases}

The present concept is considered to be an additional mechanism for cancer metastasis originated from the previous mentioned scientific background information. The concept is based on the idea that, the primary tumor mass is considered to be an endocrine gland like structure. Therefore, angiogenesis must occur as prerequisite for metastases. The hormones of this initiated pathogenic gland are specific growth factors mainly VEGF, TGF- $\alpha$ and TGF- $\beta$. The latter growth factors signaling pathways could induce tumor angiogenesis and subsequent neoplastic transformation in the stem cells localized in the different organs that possess the appropriate growth factor receptors. In another word, it is not necessary for cancer cells to migrate via blood or lymphatic vessels but, they just secrete their hormone like action; transforming growth factors into the blood stream according to endocrine system so as to exert malignant transformation in the somatic stem cells , as a consequence of their multiple excessive exposures to these specific Biomolecules released from the primary malignant cells. The outcome of this event is a formation of secondary tumor without the necessity of migration of the primary cancer cells. This concept may explain the occurrence of secondary tumors with unknown primary, the condition could be sometimes observed by the clinical oncologist during cancer management (Figure 5).

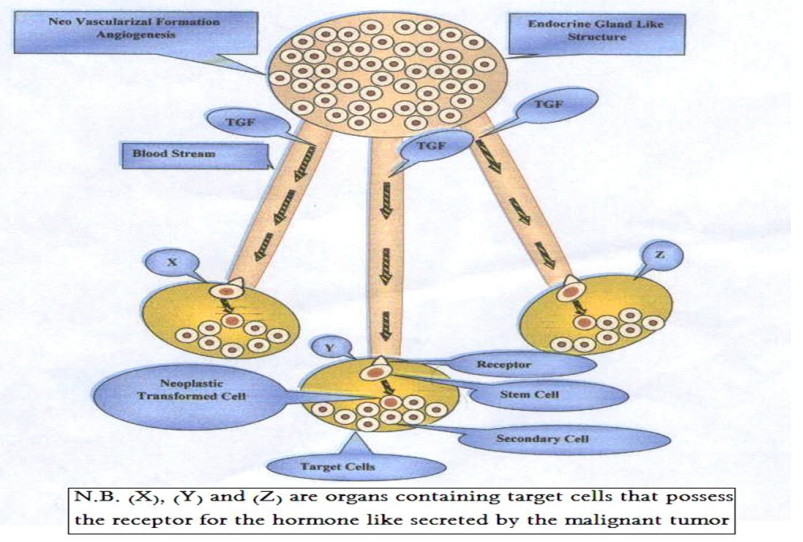

Figure 5 Diagrammatic representation of the novel concept for cancer metastasis. 


\section{Conclusion}

i. Growth factors (GFs) presented by VEGF, TGF- $\alpha$ and TGF- $\beta$ could play crucial role in enhanced cancer metastasis.

ii. Somatic stem cells that are localized in the different organs could be the site of neoplastic transformation.

iii. The previous mentioned GFs and stem cells could co-operate to enhance cancer metastasis.

iv. The clinical impact of the present concept could lead to design a targeted biological therapy so as to stop cancer metastases.

\section{Acknowledgements}

None.

\section{Conflict of interest}

The author declares no conflict of interest.

\section{References}

1. Waterfield MD. Epidermal growth factor and related molecules. Lancet. 1989;1(8649):1243-12456.

2. Garden Y, Ullrich A. Molecular analysis of signal transduction by growth factors. Biochemistry. 1988;27(9):3113-3119.

3. Sporn MB, Robert AB. Peptide growth factors are multi-functional Nature. 1988;332(6161):217-219.

4. BAuthorBianco R, Melisi D, Ciardiello F, et al. Key cancer cell signal transduction pathways as therapeutic targets. Eur $J$ Cancer. 2006;42(3):290-294.

5. Sasi W, Sharma AK, Mokbel K. The Role of suppressors of cytokine signalling in human neoplasms. Mol Biol Int. 2014;2014:630797.

6. Coleman SJ, Bruce C, Chioni AM, et al. The ins and outs of fibroblast growth factor receptor signalling. Clin Sci (Lond). 2014;127(4):217-231.

7. Mo JS, Park HW, Guan KL. The Hippo signaling pathway in stem cell biology and cancer. EMBO Rep. 2014;15(6):642-656.

8. Holley RW. Control of growth of mammalian cells in culture. Nature. 1975;258(5535):487-490.

9. Aaronson SA. Growth factors and cancer. Science. 1991;254(5035):11461153.

10. Witsch E, Sela M, Yarden Y. Roles for growth factors in cancer progression. Physiology (Bethesda). 2010;25(2):85-101.

11. Lippman ME, Dickson RB, Bates S, et al. $8^{\text {th }}$ San Antonio Breast Cancer Symposium -Plenary lecture. Autocrine and paracrine growth regulation of human breast cancer. Breast Cancer Res Treat. 1986;7(2):59-70.

12. Sporn MB, Roberts AB. Autocrine growth factors and cancer. Nature. 1985;313(6005):745-747.

13. Roberts B, Anzano MA, Wakefield LM, et al. Type beta transforming growth factor: a bifunctional regulator of cellular growth. Proc Natl Acad Sci USA. 1985;82(1):119-123.

14. Kimchi A, Wang X-F, Weinberg RA, et al. Absence of TGF-beta receptors and growth inhibitory responses in retinoblastoma cells. Science. 1988;240(4849):196-199.

15. Kowanetz M, Ferrara N. Vascular endothelial growth factor signaling pathways: therapeutic perspective. Clin Cancer Res. 2006;12(17):50185022 .

16. Yilmaz M, Christofori G. EMT, the cytoskeleton, and cancer cell invasion. Cancer Metastasis Rev. 2009;28(1-2):15-33.
17. Wu Y, Hooper AT, Zhong Z, et al. The vascular endothelial growth factor receptor (VEGFR-1) supports growth and survival of human breast carcinoma. Int J Cancer. 2006;119(7):1519-1529.

18. Jura N, Endres NF, Engel K, et al. Mechanism for activation of the EGF receptor catalytic domain by the juxtamembrane segment. Cell. 2009;137(7):1293-1307.

19. BAuthor\%Brussino L, Culla B, Bucca C, et al. Inflammatory cytokines and VEGF measured in exhaled breath condensate are correlated with tumor mass in non-small cell lung cancer. J Breath Res. 2014;8(2):027110.

20. Folkman J. Angiogenesis: an organizing principle for drug discovery? Nat Rev Drug Discov. 2007;6(4):273-286.

21. BAuthor\%BAuthor\%Bonnesen B, Pappot $\mathrm{H}$, Holmstav J, et al. Vascular endothelial growth factor A and vascular endothelial growth factor receptor 2 expression in non-small cell lung cancer patients: relation to prognosis. Lung Cancer. 2009;66(3):314-318.

22. BAuthor\%Carrillo de Santa Pau E, Arias FC, Caso Pelaez E, et al Prognostic significance of the expression of vascular endothelial growth factors A, B, C, and D and their receptors R1, R2, and R3 in patients with nonsmall cell lung cancer. Cancer. 2009;115(8):1701-1712.

23. Ito $\mathrm{K}$, Suda $\mathrm{T}$. Metabolic requirements for the maintenance of selfrenewing stem cells. Nat Rev Mol Cell Biol. 2014;15(4):243-256.

24. Zizzino A, Ruff E, Rizzino H. Induction and modulation of anchorageindependent growth by Platelet-derived growth factor, fibroblast growth factor and transforming growth factor-beta. Cancer Res. 1986;46(6):2816-2820.

25. McGeady ML, Kerby S, Shankar V, $t$ al. Infection with a TGF-alpha retroviral vector transforms normal mouse mammary epithelial cells but not normal rat fibroblasts. Oncogene. 1989;4(11):1375-1382.

26. Finzi E, Fleming T, Segatto O, et al. The Transforming Growth Factor type -alpha coding sequence is not a direct-acting oncogene when over expressed in NIH 3T3 cells. Proc Natl Acad Sci USA. 1987;84(11):37333737.

27. Ciardiello F, McGeady ML, Kim N, et al. Transforming growth factor -alpha expression is enhanced in human mammary epithelial cells transformed by an activate $\mathrm{c}-\mathrm{Ha}$-ras Protooncogene but not by the c-neu protooncogene, an Over expression of the transforming growth factor-alpha complementary DNA leads to Transformation. Cell Growth Differ. 1990;1(9):407-420.

28. Rosenthal A, Lindquist PB, Bringman TS, et al. Expression in rat fibroblasts of a human transforming growth Factor-alpha cDNA results in transformation. Cell. 1986;46(2):301-309.

29. Tang B, Zhu B, Liang Y, et al. Asiaticoside suppresses collagen expression and TGF- $\beta /$ Smad signaling through inducing Smad 7 and inhibiting TGF- $\beta$ RI and TGF- $\beta$ RII in keloid fibroblasts. Arch Dermatol Res. 2011;303(8):563-572.

30. Chen HY, Huang XR, Wang W, et al. The Protective Role of Smad7 in Diabetic Kidney Disease: Mechanism and Therapeutic Potential. Diabetes. 2011;60(2):590-601.

31. Yamaguchi T, Matsuzaki K, Inokuchi R, et al. Phosphorylated Smad2 and Smad3 signaling: Shifting between tumor suppression and fibrocarcinogenesis in chronic hepatitis C. Hepatol Res. 2013;43(12):13271342.

32. Matsuzaki K. Smad phospho-isoforms direct context-dependent TGF- $\beta$ signaling. Cytokine Growth Factor Rev. 2013;24(4):385-399.

33. Lan HY. Diverse roles of TGF- $\beta /$ Smads in renal fibrosis and inflammation. Int J Biol Sci. 2011;7(7):1056-1067.

34. Marone M, Scambia G, Bonanno G, et al. Transforming growth factor beta 1 transcriptionally activates CD34 and prevents induced differentiation of TF-1 cells in the absence of any cell cycle effects. Leukemia. 2002;16(1):94-105. 
35. Monier MN, Fortnel N, Ducos K, et al. TGF-(beta) 1 maintain hematopoietic immaturity by a reversible negative control of cell cycle and induces CD34 antigen up modulation bone-metastasizing cancer cells. Clin Exp Metastasis. 1999;17:27.

36. Wojtowicz Praga S. Reversal of tumor-induced immuno suppression: a new approach to Cancer therapy. J Immunother. 1997;20(3):165-177.

37. Massague J. How cells read TGF-beta signals. Nat Rev Mol Cell Biol. 2000;1(3):169-178.

38. $\mathrm{Wu} \mathrm{K}$, Ding $\mathrm{J}$, Chen $\mathrm{C}$, et al. Hepatic transforming growth factor Beta gives rise to Tumor-initiating cells and promotes liver cancer Development. Hepatology. 2012;56(6):2255-2267.

39. Itoh $\mathrm{S}$, Itoh $\mathrm{F}$, Goumans $\mathrm{MJ}$, et al. Signaling of transforming growth factor-beta family members through Smad proteins. Eur J Biochem. 2000;267(24):6954-6967.

40. BAuthor\%Hiemer SE, Varelas X. Stem cell regulation by the Hippo pathway. Biochim Biophys Acta. 2013;1830(2):2323-2334.

41. Reya T, Morrison SJ, Clarke MF, et al. Stem cells, cancer, and cancer stem cells. Nature. 2001;414(6859):105-111.
42. BAuthor\%Tu LC, Foltz G, Lin E, et al. Targeting stem cells-clinical implications for cancer therapy. Curr Stem Cell Res Ther. 2009;4(2):147153

43. Lobo NA, Shimono Y, Qian D, et al. The biology of cancer stem cells Annu Rev Cell Dev Biol. 2007;23:675-699.

44. Owens DM, Watt FM. Contribution of stem cells and differentiated cells to epidermal tumours. Nat Rev Cancer. 2003;3(6):444-451.

45. Fidler IJ, Balch CM. The biology of cancer metastasis and implication for therapy. Curr Probl Surg. 1987;24(3):129-209.

46. BAuthor\%Tanaka N, Kikuchi E, Kanao K, et al. Metastatic behavior of upper tract urothelial carcinoma after radical nephroureterectomy: association with primarytumor location. Ann Surg Oncol. 2014;21(3):1038-1045.

47. Miller FR, Heppner GH. Cellular interaction in metstasis. Cancer metastasis Rev. 1990;9(1):21-34. 